\title{
EDUCACIÓN E INTERCULTURALIDAD PERSPECTIVAS TEÓRICAS Y PRACTICAS EN AMÉRICA LATINA
}

\author{
EDUCAÇÃO E INTERCULTURALIDADE \\ PERSPECTIVAS TEÓRICAS E PRÁTICAS NA AMÉRICA LATINA \\ EDUCATION AND INTERCULTURALITY \\ THEORETICAL AND PRACTICAL PERSPECTIVES IN LATIN AMERICA
}

Raoni Machado Moraes Jardim

E-mail: raoni.mmj@gmail.com

Doctorado por el Departamento de Estudos Latinoamericanos de la Universidad de Brasília

$(\mathrm{ELA} / \mathrm{UnB})$

Máncel Martínez Ramos

E-mail: mmancel@yahoo.com.mx

Doctorado en Ciencias Sociales y Humanas de la Pontificia Universidad Javeriana de Bogotá

\section{RESÚMEN}

¿Cuáles son los sentidos de la interculturalidad? ¿Cómo se ha utilizado este concepto en el campo educativo? ¿Cuáles perspectivas teóricas y prácticas se vienen desarrollando a partir de él? ¿Por qué se ubica como un paradigma para la formulación de políticas públicas, educativas y de investigación? ¿Por qué es importante para la lucha por el reconocimiento de epistemes subalertinazadas? ¿Cuáles son sus riesgos, conquistas y desafíos? La interculturalidad despierta muchas preguntas. En este artículo se abordan algunas de ellas con el interés de colaborar con el debate sobre la interculturalidad en la docencia, la investigación y la producción de conocimiento en Latinoamérica. Se problematiza la posición "de dominador-dominado" que los docentes de la región asumen o rechazan dentro de la actual estructura de producción de conocimiento, fuertemente condicionada por actores neoliberales. El pensamiento decolonial se ubica como un campo importante para la aplicación de eses análisis o simplemente para analizar la perpetuación, la reacomodación o el rompimiento con las herencias coloniales rumbo a una universidad más abierta y representativa de las comunidades y epistemes latinoamericanas, incluyendo los conocimientos de las comunidades tradicionales. Los sentidos de la interculturalidad ganan correspondencia con el "grado" del llamado giro epistémico dentro de las propuestas de diferentes pensadores de este tema, que es crucial para la transformación de realidades.

PALABRAS-CLAVE: Educación Intercultural. Saberes Tradicionales. Pensamiento Decolonial.

\section{RESUMO}

Quais são os sentidos de interculturalidade? Como esse conceito tem sido utilizado no campo educacional? Quais perspectivas teóricas e práticas vêm sendo desenvolvidas a partir dele? Por que se coloca como um paradigma para a formulação de políticas públicas educativas e de pesquisa, e para a luta por reconhecimento de epistemes subalternizadas? Quais são seus riscos, suas conquistas e desafios? A interculturalidade desperta muitas perguntas. $O$ artigo proposto aborda algumas destas questões, colaborando com o debate sobre a interculturalidade na docência, na pesquisa e na produção de conhecimento no ensino superior latinoamericano. Almeja-se problematizar, nesse processo, a posição "dominador-dominado" que os docentes da região assumem, ou rejeitam, dentro da atual estrutura de produção de conhecimento, fortemente condicionada por atores neoliberais. O Pensamento Decolonial é colocado como um importante campo para aplicação dessa análise sobre as perpetuações, recolocações e rompimentos com as heranças coloniais rumo a uma universidade mais aberta e representativa das comunidades e epistemes latino-americanas, com a inclusão das comunidades tradicionais. Os sentidos da interculturalidade ganham correspondência com o "grau" do chamado giro epistêmico dentro das propostas de diferentes pensadores desse tema tão crucial para a transformação de realidades.

PALAVRAS-CHAVE: Educação intercultural. Saberes tradicionais. Pensamento decolonial. 
ABSTRACT

What are the meanings of interculturality? How has this concept been used in the educational field? What theoretical and practical perspectives have been developed from it? Why is interculturality a paradigm for the formulation of public educational and research policies, and for the struggle for the recognition of subalternized epistemes? What are its risks, its achievements and challenges?

Interculturality raises many questions. The proposed article addresses some of these issues, collaborating with the debate on interculturality in teaching, research and knowledge production in Latin American higher education. It is hoped to problematize, in this process, the dominator-dominated position that the teachers of the region assume, or reject, within the current structure of production of knowledge, strongly conditioned by neoliberal actors. Decolonial Thinking is an important field for the application of this analysis on the perpetuations, replacements and disruptions with colonial inheritances towards a more open and representative university of Latin American communities and epistemes, with the inclusion of traditional communities. The meanings of interculturality correspond to the "degree" of the so-called epistemic turn within the proposals of different thinkers of this theme so crucial to the transformation of realities.

KEYWORDS: Intercultural Education. Traditional Knowledge. Decolonial Thinking.

\section{INTERCULTURALIDAD - APROXIMACIONES AL CONCEPTO}

La interculturalidad, expresada como "educación intercultural", es evocada crecientemente por autores y corrientes académicas para problematizar los límites y las consecuencias del pensamiento moderno colonial en América Latina, buscando construir conocimientos a partir del diálogo con ricas y diversas epistemes no hegemónicas.

La bibliografía revela multiplicidad de significados y de propuestas prácticas y teóricas para combatir las herencias del colonialismo en el campo académico y desarrollar un pensamiento social más representativo de las comunidades latinoamericanas diversas.

Es necesario reconocer la pluralidad de significados que tiene la interculturalidad, incluso si sólo consideramos el ámbito educativo. Una primera aproximación a la interculturalidad apunta hacia la flexibilidad del concepto de "cultura", en general reconocida en cuanto valores y conocimientos compartidos entre sujetos, vinculados a un territorio y a unas prácticas, junto con el dinamismo de sus contactos.

Pensemos en las diferencias culturales entre dos vecinos que viven en Brasilia. Uno nació y fue criado en el Plano Piloto, en un barrio privilegiado; el otro nació en una comunidad rural de Planaltina, ciudad cercana a Brasilia con gran parte de la población con bajos ingresos. Sus "diferencias culturales" pueden parecer menores al compararlas con las que hay entre un ejecutivo de São Paulo y un indígena Yanomami. Al principio, esos vecinos pueden ser clasificados como occidentales, modernos, latinoamericanos, urbanos y brasileños. Imaginemos que la familia de Planaltina llegó al DF junto con otras familias del nordeste 
brasileño, componiendo un sector habitacional específico dentro de la comunidad rural. De origen marañense, los padres aprendieron una técnica de plantación específica, además de tener costumbres y valores diversos, son responsables, dentro de la economía local, por la producción de mandioca. Al llegar a Brasilia, ese sujeto conoció un grupo evangélico y se hizo practicante. Entró en la universidad donde conoció a su novia, integrante de un terreiro de umbanda. En el altar de la sala tiene santos católicos junto a estatuas de orixás, regalados por su novia, y un patuá indígena que su madre, indígena Guajajara, le dio.

Pensemos en un pueblo supuestamente más homogéneo, como los indígenas Yanomamis, que suelen tener la imagen de una cultura más cerrada y estática para quien observa de lejos. Para quien está en la Amazonía o conoce sus particularidades, los pueblos Yanomamis son muy diversos y varían culturalmente de acuerdo con el grupo y territorio. Son también pueblos que viven y transitan fronteras internacionales, teniendo gran contacto con colombianos y venezolanos, indígenas y no indígenas. Muchos de los Yanomamis están en lucha, resistencia o bajo la influencia constante de madereros, garimpeiros, megaproyectos, agronegocios extensivos, grupos armados, narcotráfico y otros procesos extractivistas que devastan la selva. Algunos pueblos tienen mayor acceso a Internet, o mayor influencia de misioneros y grupos religiosos, etc.

Independientemente del concepto de cultura que se adopte, las características culturales son diversas y su dinamismo crece cuanto se observa con más profundidad el trayecto desde lo social hacia lo comunitario, lo familiar y lo individual. Ciertamente el factor territorial y la transmisión generacional de los valores y conocimientos agregan un sentido más permanente. Pero, además del dinamismo cultural dentro de un territorio, quien permanece es "visitado por otras culturas". Los sujetos pueden moverse y llevar con ellos aquella "cultura" y encontrar otros sujetos, también en movimiento. Estos intercambios ocurren todo el tiempo.

Incluso en las poblaciones más aisladas (expresión que, en general, toma como referencia sociedades urbanas), la construcción identitaria y cultural es impulsada por momentos de contacto con individuos de otras culturas. La alteridad es parte fundamental de la construcción cognitiva y emocional de la identidad grupal e individual. Sólo es posible definirse en contraposición a un "otro"; los contactos entre humanos, e incluso otros tipos de contactos, nos modifican permanentemente al mismo tiempo que ayudan a definirnos. 
Aunque el desarrollo de las culturas sea un fenómeno intercultural, existen núcleos que mantienen características similares. La afirmación de esos rasgos identitarios, étnicos y raciales es fundamental para el reconocimiento, respeto y fortalecimiento de las luchas de las comunidades en procura de justicia social e histórica. Es importante considerar también que los encuentros no son simétricos. Hay configuraciones culturales que ejercen mayor peso e influencia, manifestándose, inclusive, como violencias simbólicas y físicas. Son vectores de poder construidos históricamente, pero que pueden ser alterados. Estos contactos pueden resultar en síntesis diversas e improbables, a menudo asimétricas.

\section{DE LA INTERCULTURALIDAD A LA EDUCACIÓN INTERCULTURAL}

En el campo educativo, el uso teórico del concepto de interculturalidad puede relacionarse con la interdisciplinariedad ${ }^{1}$. En la conformación de las disciplinas, la propuesta cartesiana de fragmentar la realidad para conocerla y dominarla, se hizo hegemónica en el campo científico y académico, impulsada por el capitalismo.

La disciplinarización del conocimiento tiene un fuerte impacto sobre las subjetividades y sus proyecciones y modelos de realidad. Pero el carácter relacional y complejo de esta realidad y sus acontecimientos, no encuentran reflejo en estas disciplinas, ya que la vida es más compleja y transdisciplinaria. Esta disciplinarización de las dimensiones de la vida es una simplificación reduccionista, propia de la racionalidad occidental hegemónica.

Es importante percibir el carácter militar que la palabra "disciplina" carga, referente a la "obediencia a reglas, superiores y reglamentos" ${ }^{2}$. De cualquier forma, la mirada disciplinar es la mirada más valorada en el pensamiento hegemónico occidental. El propio uso del término "interdisciplinar" denota nuestra clave disciplinar.

Aunque los límites paradigmáticos de la disciplinarización del conocimiento parecen aproximarse y que las ciencias sociales latinoamericanas vengan insistiendo en la importancia que la interculturalidad y las epistemes no-disciplinares tienen como paradigma de producción de conocimiento, es necesario percibir la distancia entre la propia praxis intercultural y la mera teorización sobre su importancia. Eso tornase aún más preocupante cuando se evoca el

${ }^{1}$ Ver CARVALHO Y FLÓREZ-FLÓREZ (2014, p.132).

${ }^{2}$ Ver https://www.priberam.pt/dlpo/disciplina. 
lugar de habla "subalterno latinoamericano", siendo este desplazamiento una violencia propia de la "colonialidad del saber ". 3

No por casualidad, los sujetos "subalternizados" son los mismos que cultivan epistemes no disciplinares. Al no despertar interés para el capitalismo, o mejor, por representar amenazas a la conformación del pensamiento hegemónico eurocéntrico, dicotómico, racista, disciplinar e jerarquizante, estos pueblos enfrentaran, y siguen enfrentando, diversas violencias. En el campo epistémico resaltamos el despojo, la apropiación, la invisibilización y la marginación de sus conocimientos. La búsqueda por naturalizar la superioridad europea dentro de la jerarquización social y del trabajo, impuso una lógica perversa en la cual el punto de vista europeo, concebido como ideal, debe ser deseado sin nunca ser alcanzado por los demás pueblos. La Iglesia Católica jugó un papel definitivo sobre esa naturalización, entendida, como un designio divino, manifiesto, incluso, biológicamente y fenotípicamente, por el color de la piel. A pesar de que la violencia se ha producido especialmente contra los pueblos indígenas y africanos, también en Europa se sufrió marginación epistémica, lo que se ilustra al comparar la filosofía espinosista ante la cartesiana, o la de Goethe frente a la newtoniana.

Puede ser que para los sujetos de matrices epistémicas de pueblos y comunidades tradicionales, donde abundan perspectivas no disciplinarias, no tenga tanto sentido hablar de interdisciplinaridad, ya que la propia disciplina no sería concebida, o no es, necesariamente, hegemónica. En general, son conocimientos aprendidos y transmitidos relacional y generacionalmente, buscando abordar aspectos de la vida, y la vida, así como las subjetividades, es "inter" y "transdisciplinar", con aspectos complejos atravesados por múltiples dimensiones. Es posible llamar a esas formas de conocimiento de diversas maneras dentro y fuera del ámbito disciplinar académico: relacional, complejo, holístico, integrado, abierto, circular, transdisciplinar, sistémico, etc, Estos son conocimiento cargados de potencia para el desarrollo de tecnologías sociales capaces de mejorar la calidad de vida de la población y el bienestar subjetivo y comunitario, vinculando los procesos abstractos del pensar con la corporeidad, la afectividad, la espiritualidad y la comprensión de la complejidad de los fenómenos.

Nada impide reconocer los logros que los conocimientos disciplinares, que dominan los campos académicos y científicos, han generado y generarán. Pero la marginación de 
perspectivas interdisciplinares, especialmente de las matrices tradicionales, trae pérdidas fundamentales, disminuyendo también la capacidad de avance de los conocimientos disciplinares. Estas pérdidas cognitivas, emocionales y de identidad, dificultan el avance hacia sociedades más justas y empobrecen a las universidades y sociedades latinoamericanas.

Pero no es suficiente inserir los conocimientos tradicionales de forma fragmentada en las esferas de producción de conocimiento, buscando apenas los beneficios unilaterales que eso traería a los paradigmas académicos en crisis. Acá volveríamos nuevamente a la “colonialidad del saber". La lógica académica es fuertemente colonial y utilitarista, así como lo son los Estados-nacionales que instrumentalizan el discurso del multiculturalismo ${ }^{4}$ volviéndolo una narrativa vigente muy útil al orden neoliberal. ${ }^{5}$ Reconocer los saberes tradicionales, antes que nada, debe partir del respeto y reconocimiento de los sujetos subalternizados, discriminados o desconocidos históricamente. Es fundamental garantizar la soberanía cultural y territorial y los derechos sociales de acuerdo a sus especificidades y diversidad. La importancia de reconocer los conocimientos tradicionales no debe estar por encima de reconocer la deuda histórica hacia los sujetos de los pueblos y comunidades tradicionales, que en la mayoría de los casos siguen despojados de sus territorios y en situación de enorme vulnerabilidad social. La interculturalidad exige de quién la evoca un comprometimiento con la praxis. Exige que el diálogo y los beneficios de estos encuentros entre culturas ocurran considerando las necesidades y los límites de ambos los lados.

En América Latina tenemos ejemplos relevantes de esta ocupación práctica de la teoría. El colombiano Fals-Borda (1991, 1982, 2002), con la Investigación Acción Participativa, articuló la producción de conocimiento con el cambio social. Él reconocía en cada comunidad sus saberes propios y los consideraba elementos claves en el diseño de las investigaciones. Esta innovación metodológica fue aplicada con sectores populares, principalmente campesinos y de proletariado agrícola, esforzándose en transformar las realidades. El trabajo de Fals-Borda (1963) incluye amplias reflexiones sobre el papel de las universidades latinoamericanas, proponiendo una universidad más participativa y actuante en la transformación social. Su trabajo se extiende en países como México, Nicaragua, Paraguay y Brasil. En el libro "E1 Brasil, campesinos y vivienda" escrito a partir de su investigación en los barrios de Palmital y Pãe Nosso, resalta que desconocer los saberes negros llevó a una atomización de las viviendas perdiendo sus espacios sociales y desconocer los saberes

${ }^{4}$ Ver ZIZEK, JAMESON E GRUNER (2008) y BOURDIEU E WACQUANT (2002).

${ }^{5}$ Ver BORÓN (2005). 
indígenas de la región, llevó al desprecio de soluciones como las casas circulares y el uso de materiales nativos.

En Brasil, autores como Darcy Ribeiro, Anísio Teixeira y Florestan Fernandes, estuvieron comprometidos con un pensamiento latinoamericano y brasileño autónomo y en ejercicio de su pluralidad cultural. Paulo Freire (1987), elaboró una fértil reflexión y práctica con los llamados grupos "subalternos", asociando las ideas de praxis con el enfoque dialógico, estableciendo las bases de la Pedagogía del Oprimido y de la Educación Libertadora, y generando una verdadera escuela de enseñanza en América Latina y en el mundo.

Mato (2014) traza un histórico sobre las obras e influencias y apunta como Freire y Borda inauguraron o influenciaron pedagogías que buscan la liberación de las directrices externas sobre la producción de conocimiento, considerando los sujetos y sus contextos de vida durante la enseñanza. Se modifica el estudio "del otro" para el estudio "con el otro", siendo éste una necesidad ética, política y epistemológica. Estas propuestas ponen en jaque las corrientes teóricas que confunden, intencionalmente o no, el contacto con grupos excluidos históricamente de la enseñanza formal, con algún giro epistémico efectivo. ${ }^{6}$

Se puede, perfectamente, tener contacto y hasta militar junto a los pueblos tradicionales, y seguir teniendo como principal resultado producciones académicas dentro de la "clave epistémica hegemónica / occidental y colonial". La propia cercanía con grupos subalternizados puede funcionar como una estrategia para acceder a informaciones privilegiadas, a ejemplo de lo que el multiculturalismo, en sus versiones instrumentales y perversas, busca. Mientras tanto, se pude seguir alimentando revistas internacionales; valorando currículos de autores; proyectando centros académicos y corrientes teóricas. En fin, una búsqueda por cambios sociales... de forma que nada se altere efectivamente. Este es el abismo que separa la propuesta del llamado giro decolonial de lo que seria un giro epistémico efectivo.

Carvalho (2010) realiza una importante reflexión sobre la "espectacularización"7 y la "canibalización"8 de las culturas populares latinoamericanas. Ante el riquísimo universo de festejos, juegos y ceremonias que, además de sagradas, son de enorme belleza "estética", el autor alerta sobre el interés mercantil de sectores del turismo y del entretenimiento

\footnotetext{
${ }^{6}$ La critica puede encontrar lastro en un análisis del discurso apliaco al texto de Castro-Gómez y Grosfoguel (2007).

${ }^{7}$ Ver Carvalho (2010, p.47).

${ }^{8}$ Ver Carvalho (2010, p.63). 
impulsados por la industria cultural con el apoyo del Estado. Es importante percibir que las comunidades tradicionales no son víctimas sólo de la industria del turismo y del entretenimiento, sino también de los académicos e intelectuales. Éstos, muchas veces, establecen relación con las comunidades de forma predatoria, dejando intencionalmente de tratar las dimensiones económicas, estéticas y políticas de esas relaciones mercadológicas. La resignificación ocurre de fuera hacia adentro, en una dinámica unilateral. ${ }^{9}$

Después de "incorporar" al "otro", el actor de la "acción caníbal" puede retirarlo de escena y se presenta como si fuera el "otro"10. Esta lógica, que parece traer influencias de una concepción antropofágica, principalmente en el contexto brasileño, no toma conciencia de las asimetrías de poder entre esa clase intelectual o artística y los artistas populares, en general pobres y marginados de diversos derechos ${ }^{11}$.

Según Mato (2006), la exclusión histórica de los saberes no científicos no puede ser resuelta por la "folklorización" de la diversidad cultural. Se necesita reconocer los conflictos contemporáneos, especialmente en las experiencias socioeducativas; y trabajar intensamente en la reconstrucción de nuestras sociedades buscando la colaboración entre diversas formas de saber. De la misma forma, ante la dificultad de construir conocimientos no apenas sobre los sujetos subalternizados, sino con ellos, se debe al menos articular su estudio con el estudio de las prácticas de agentes transnacionales de carácter neoliberal, tales como el Banco Mundial y la Agencia Internacional de los Estados Unidos para el Desarrollo, para percibir las articulaciones de poder que conectan a esos actores hegemónicos con los locales.

\section{TEORÍAS Y PRÁCTICAS DE LA EDUCACIÓN INTERCULTURAL EN AMÉRICA LATINA}

Los currículos monoepistémicos y eurocentrados y la exclusión de narrativas y de los propios sujetos con epistemologías diversas, no representan la riqueza cultural de los países de la región y reflejan un proyecto de poder hegemónico racista vigente desde la colonización. Transformar esa realidad pasa por las disputas de proyectos de poder y por los actores que enuncian y componen las narrativas oficiales e históricas.

\footnotetext{
${ }^{9}$ Ver Carvalho (2010, p.48).

${ }^{10}$ Ver CARVALHO (2010, p.68).

${ }^{11}$ Ver CARVALHO (2010, p.67). 
Entre los acontecimientos que impactaron la discusión sobre la educación intercultural en América Latina, destacamos la participación efectiva de los movimientos indígenas en el establecimiento de Estados Plurinacionales y la formulación de las constituciones nacionales en Bolivia, y en Ecuador con el Sumak Kaway (Buen Vivir). Son avances constitucionales, aunque no son garantía de que esas políticas sean cumplidas adecuadamente ni de que no sean canibalizadas por el neoliberalismo, como apunta Cusicanqui (2010).

Otra transformación efectiva de la educación intercultural es el establecimiento de la política de cuotas étnicas y raciales, ${ }^{12}$ adoptada en Brasil a partir de $2002^{13}$, y sancionada como la Ley $12711 / 12^{14}$ y la Ley $11645 / 08$, que determina la obligatoriedad de temática historia y cultura afrobrasileña e indígena en la formación escolar de los jóvenes. Esta política de acción afirmativa busca revertir el racismo histórico como patrón social de desigualdad, injusticia, crueldad y discriminación. Aunque las universidades aún tienen un perfil mayoritariamente blanco, el porcentaje de negros ingresan y se gradúan viene aumentando sustancialmente, casi duplicándose entre 2005 y 2015. ${ }^{15}$

La ampliación de la oferta de universidades y facultades, asociada a programas gubernamentales de financiamiento estudiantil y la política de cuotas para personas de bajos ingresos, negros e indígenas, alteró sustancialmente el perfil étnico-racial de la enseñanza superior en Brasil. La presencia de estos sujetos en la Universidad evidenció aún más el racismo epistémico, que se refiere a la perpetuación de los currículos y metodologías centrados en tradiciones europeas o estadounidenses. Nuestras narrativas siguieron referenciadas, en mayor parte, en la historia de los colonizadores y del imperio económico. La exclusividad de la cultura científica, dentro de los moldes capitalistas inaugurados por el colonialismo en las Américas, se refleja hoy en idiomas, referencias bibliográficas, sistemas de evaluación, modelos pedagógicos, disciplinarización de los conocimientos y en las exigencias a docentes e investigadores (BORÓN, 2005). Los sujetos con epistemes distintas siguen teniendo inmensa dificultad en capacitarse en la enseñanza formal y en llevar al público sus narrativas y saberes sobre la historia y sobre el mundo.

Ante este escenario, diversos autores y autoras empiezan a desarrollar el concepto de educación intercultural. Inicialmente, el término se refiere más a la educación indígena y

\footnotetext{
${ }^{12}$ Sobre el orígen de este termo en el contexto mundial, ver Carvalho, Flórez y Marttínez (2017).

13 Sobre la trayectória de esta política en Brasil, ver Carvalho (2004 y 2010).

14 Ver http://www.planalto.gov.br/ccivil 03/_ato2011-2014/2012/lei/112711.htm.

${ }^{15}$ Ver IBGE (2016). 
bilingüe, pero pronto comienza a expandirse hacia otras poblaciones y significados. Así, también en el campo educativo, la interculturalidad puede tener distintos usos, desde programas monoepistémicos para un grupo de alumnos de poblaciones tradicionales, hasta programas pluriepistémicos para alumnos de un mismo origen cultural.

Diversos autores latinoamericanos teorizan sobre la interculturalidad y su relación con la construcción de conocimiento. Hacen contribuciones y críticas o avanzan en las prácticas interculturales, con nombres y propuestas diferentes. Entre ellas y ellos están Vera María Candau, Daniel Mato, José Jorge de Carvalho, Gersen Baniwa y Antônio Bispo dos Santos, en Brasil; Fidel Tubino en Perú; Catherine Walsh, en Ecuador; Arturo Escobar, Luis Alberto Artunduaga y Celmira Castro Suárez, en Colombia; Sílvia Rivera Consicanqui, en Bolivia; Gunther Dietz, en México; Bell Hooks, en Estados Unidos, citando sólo algunos entre muchos nombres relevantes.

Candau (2009) traza un resumen histórico de la educación intercultural en América Latina. El primer período tratado, se extiende desde la colonización hasta el inicio del siglo $\mathrm{XX}$, caracterizado por la radical negación y exterminio de cualquier forma de conocimiento que no parta de los colonizadores, el epistemicídio del "otro" que consolida el pensamiento hegemónico capitalista moderno. A partir de entonces, hasta la década de 1970, ocurrió el asimilacionismo, que inicia el uso del bilingüismo como etapa para la "conversión" indígena a los preceptos educativos y ciudadanos oficiales. En la década de 1980 y con el apoyo de universidades y sectores progresistas de la Iglesia Católica, el discurso sobre el derecho a la educación indígena dentro de sus propios modos gana proyección. A continuación, ocurrió la unificación de la pauta educativa entre los pueblos indígenas y la defensa de un sistema de enseñanza propio, con docentes y cuadros directivos indígenas.

Candau (2009) reafirma la importancia del movimiento negro en la lucha por la educación intercultural, sea por la denuncia de contenidos racistas, disfrazados de mestizaje o democracia racial; la lucha por el acceso, la permanencia y la cualificación en la enseñanza; o la lucha por inserción de contenidos afro-diaspóricos en los currículos.

Como otro importante aporte hacia la interculturalidad, podemos citar la educación popular. Aunque sea identificada con el contexto extraescolar, su influencia llega hasta la enseñanza formal, revolucionando la didáctica a través de la contextualización del conocimiento y de la horizontalización de la relación entre profesor y alumno. 
Más allá de una retomada del trayecto histórico de la educación intercultural, nos interesa analizar críticamente aquellos autores que buscan lanzar sentidos sobre este término, siendo muchas veces adoptados de forma acrítica por la población académica y no académica. Esta problematización forma parte de una crítica más amplia al Pensamiento Decolonial y al Grupo Modernidad/Colonialidad ${ }^{16}$. En seguida buscaremos revelar otras concepciones que consideramos dialogan con el giro epistémico en una dimensión más radical de lucha por el diálogo horizontal entre los saberes científicos y los saberes tradicionales.

Catherine Walsh se ha consolidado como referencia sobre educación intercultural. En "Interculturalidad y colonialidad del poder: un pensamiento y posicionamiento "otro" desde la diferencia colonial", Walsh (2007) hace diferencia entre interculturalidad y multiculturalismo, reclamando para el primero el antídoto para el segundo. ${ }^{17}$ En el texto, la autora discurre sobre la confusión hecha entre ambos y el uso nocivo que el Estado hace del multiculturalismo, especialmente al confundirlo con el término interculturalidad. ${ }^{18}$ Este aporte es de gran relevancia porque revela los usos instrumentalizados que el Estado, especialmente influenciado por directrices neoliberales, hace de la interculturalidad.

Es importante notar, que la autora restringe su contribución al presentar el concepto de interculturalidad de forma genérica, lo que abre espacio, justamente, para o su manoseo, facilitando la apropiación indebida que ella critica ${ }^{19}$. Al relacionar la interculturalidad con "conocimientos otros", sin claridad en una referencia poblacional o geográfica, se abre espacio a su uso instrumental por parte de actores neoliberales multiculturalistas, por ejemplo. A pesar de que el concepto de interculturalidad es conocido por haber sido formulado fuera del contexto académico, es importante recordar que el movimiento indígena ecuatoriano estuvo en contacto con la academia al momento de la formulación del concepto y de su inclusión en la Constitución de 2008. Pero para Walsh (2007), este proceso da al término una connotación opuesta a la dinámica colonial. ${ }^{20}$

La mirada que Walsh da al campo de la filosofía cuando lleva la crítica al multiculturalismo hacia el ámbito de la producción de conocimiento, también es pertinente

\footnotetext{
${ }^{16}$ Esta critica fue desarrollada em la tesis de doctorado "Educação e o Projeto Encontro de Saberes: do Giro Decolonial ao Efetivo Giro Epistêmico", de Raoni Machado Moraes Jardim.

${ }^{17}$ Ver Walsh (2007, p.53/4).

${ }^{18}$ Ver Walsh (2002a:26. Apud WALSH, 2007, p.56)

${ }^{19}$ Ver la definición del término en Walsh (2007, p. 47).

20 Ver Walsh (2007, p.48).
} 
para el Pensamiento Decolonial. ${ }^{21}$ El concepto puesto de esta forma refuerza la idea de antagonismo entre el movimiento indígena y la academia. Sugiere que ese movimiento representa un pensamiento fuera del legado colonial.

Para evitar un exceso de dicotomías se puede problematizar la visión siempre negativa que Walsh lanza al Estado. Hay ejemplos concretos de políticas públicas brasileñas que pueden ser consideradas como triunfos de los movimientos sociales, como en el caso de las cuotas étnico-raciales. Ahora bien, el Estado brasileño no perdió su carácter neoliberal por implementar tales políticas. Walsh (2012), discurre sobre la importancia paradigmática que las nuevas constituciones de Ecuador y Bolivia inauguraron en las ciencias, el derecho y la educación. A pesar de ser resultados de la acción articulada de movimientos de la sociedad civil, especialmente indígenas, esos avances legales, y constitucionales, no impiden que los Estados sigan directrices neoliberales.

Los avances de la pauta "intercultural" en los estados nacionales no son lineales ni homogéneos. La conquista de algunas políticas, o la ocupación de algún cargo estratégico en la gestión pública por sujetos de los pueblos tradicionales, pueden ser avances y también pueden estar rodeados por apropiaciones de discurso, por maniobras y cooptaciones de actores hegemónicos. Walsh (2012) desarrolla el concepto, y lanza tres posibilidades de interculturalidad: la relacional, la funcional y la crítica; que también son analizadas por Tubino.

La interculturalidad relacional, se refiere al contacto e intercambio entre culturas, es decir, entre personas, prácticas, saberes, valores y tradiciones culturales distintas, lo que podría darse en condiciones de igualdad o desigualdad. Para la autora ese es un tipo de interculturalidad que siempre ocurrió en el continente sudamericano ${ }^{22}$. En la visión de la autora, sin embargo, tal perspectiva es ineficaz, ya que oculta o minimiza, la conflictividad y los contextos de poder en los que las relaciones interculturales ocurren.

La interculturalidad funcional, se asemeja al multiculturalismo, característico del neoliberalismo estadounidense, acercándose también al "interculturalismo europeo", 23 en la medida en que propone reconocer la diversidad cultural como meta a ser trabajada por la vía de la inclusión en la estructura social hegemónica, sin preocupación por la alteración de esas

\footnotetext{
${ }^{21}$ Ver Walsh (2007, p.55/6).

${ }^{22}$ Ver Walsh (2012, p.63).

${ }^{23}$ Ver Walsh (2012, p.64). 
estructuras, que siguen la lógica neoliberal-individualista, pero agregando narrativas de "humanización" y "diversidad ". Esta forma, al tiempo que busca la inclusión de esos actores dentro del sistema capitalista mundializado, neutraliza a sus potenciales contestatarios. ${ }^{24} \mathrm{La}$ interculturalidad funcional sería entonces una estrategia adoptada por Estados nacionales, y por organismos internacionales y transnacionales con fuerte actuación sobre América Latina (UNESCO, PNUD, BID, CEPAL, BM, FMI, etc). Cusicanqui (2010) analiza a fondo ese tipo de interculturalidad y sus efectos, especialmente en la realidad boliviana y en los discursos del Estado con relación al etnoturismo, así como su presencia en discursos académicos, en las penetraciones del neoliberalismo en las ciencias sociales, incluso en campos con intenciones contrahegemónicas, como el pensamiento decolonial.

La interculturalidad crítica, con la que Walsh busca alinearse, significa, al mismo tiempo, el contacto cultural dialógico y la inclusión de una perspectiva crítica, de transformación de la realidad capitalista ${ }^{25}$.

Tomando el enfoque de Walsh, en este trabajo aproximamos el concepto de interculturalidad al de interculturalidad relacional. El multiculturalismo al concepto de interculturalidad funcional. Y la transculturalidad crítica, sería aproximada a la interculturalidad crítica.

Pensamos que hay una laguna en este vocabulario para expresar las experiencias positivas de la interculturalidad relacional o las experiencias negativas de la interculturalidad crítica, lo que exige hablar con aproximaciones. Para Walsh (2012), la interculturalidad relacional es negativa, ya que deja de lado la conflictividad y los contextos de poder en los que las relaciones interculturales ocurren. Pero, ese nivel relacional de la interculturalidad puede ser admitido como un acontecimiento cotidiano, fundamental de la formación identitaria, de las relaciones afectivas, de la comunicación, y en fin, de la dinámica humana. En una situación de encuentro entre sujetos de diferentes matrices culturales, se puede concebir el acontecimiento como valioso para todos sin que sea posible agotar las cuestiones estructurales que los envuelven y sin, necesariamente, resultar en "conocimientos otros". Por lo tanto, la forma negativa como esa autora concibe esa naturaleza de interculturalidad, sea por no tener un carácter crítico o por tener un carácter de deformación de las culturas en contacto, no es aquí adoptada. 
Sobre las experiencias de interculturalidad funcional, existen movimientos de conquista y retroceso con relación al Estado ya que las políticas interculturales no siguen flujos lineales. La visión homogénea y dicotómica sobre la negatividad del Estado y sobre la positividad de un proyecto de interculturalidad ¿hasta donde colabora y hasta donde entorpece la modificación de las realidades de desigualdad social? El Estado, incluso de forma parcial y fragmentada, puede contribuir a diversas luchas en el campo étnico-racial. Brasil con las cuotas raciales y también Bolivia y Ecuador, con las nuevas constituciones nacionales, que Walsh (2012) reconoce como un avance paradigmático, son ejemplos de eso. La visión homogeneizante y dicotómica del Estado y de las luchas sociales, frecuente en algunos pensadores decoloniales suele ocultar complejidades fundamentales.

Las luchas de los pueblos y comunidades tradicionales latinoamericanas, como nos alerta Cusicanqui (2010), no necesariamente se plantean contra el proyecto de modernidad o contra el sistema capitalista. A menudo, son luchas por la inclusión calificada en ese mismo sistema, o son luchas por la supervivencia en el presente, en el pasado como memoria y en el futuro como pueblos con reconocimiento y autodeterminación. La cuestión de clase y la problematización de un sistema basado en la desigualdad social, sí es algo que nos parece transversal en estas luchas.

En la interculturalidad crítica, el adjetivo "crítico" hace referencia a la conjugación de una determinado estudio o diálogo que incluye pero está más allá de las teorías críticas al capitalismo, reconociendo siempre las desigualdades e injusticias sociales. En general, cuando ese término aparece, suele ser relacionado con la Teoría Crítica inaugurada por Max Horkheimer y practicada por los miembros de la Escuela de Frankfurt, en la que el marxismo está presente con fuerza, a través de aplicaciones y relecturas diversas ${ }^{26}$.

Walsh (2012), tras ofrecer la solución a las injusticias a través del "proyecto interculturalidad crítica", afirma que esa interculturalidad parte de los segmentos subalternizados históricamente ${ }^{27}$, y alinea su propuesta a la decolonialidad ${ }^{28}$. La difusión de la idea de decolonialidad como solución para los males que persistieran desde la colonización es algo presente en muchos textos de autores oriundos del Grupo Modernidad/Colonialidad ${ }^{29}$. Walsh adopta binarismos para situar la interculturalidad crítica, como también hace gran parte

\footnotetext{
${ }^{26}$ Ver Payne (2008, p. 613 e 173, respectivamente).

${ }^{27}$ Ver Walsh (2012, p.65).

${ }^{28}$ Ver Walsh (2012, p.62 y 69).

${ }^{29}$ Ver, por ejemplo, Castro-Gómez y Grosfoguel (2007, p.17). 
del pensamiento moderno occidental hegemónico, criticado por los decoloniales, en formulaciones en torno a la "colonialidad del saber".

Nos parece desafortunado el automatismo de la asociación entre "crítico" y "decolonial". La dimensión crítica, como tradición teórica que busca comprender las dinámicas de clase, se muestra precaria en la producción decolonial ${ }^{30}$, para quien realiza una lectura sistemática de los escritos decoloniales. Aún se necesita incorporar decididamente una dimensión analítica de clase, esto es fundamental para las luchas de los grupos diversos dentro del capitalismo, pero no asegura que un sistema no capitalista garantice el protagonismo a estos sujetos.

Tomando como universo de análisis la educación: ¿Educación intercultural es un programa con estudiantes de varios segmentos culturales? ¿Un programa que incorpora literaturas y autores sobre los diferentes grupos? ¿Son los maestros y sabedores de las culturas siendo los profesores en las universidades? La interculturalidad tiene niveles diferentes.

Nuestra visión problematizadora de los términos propone un estado de alerta sobre significados y prácticas, detallando lo que se quiere decir o crear cuando son invocados: ¿Esa interculturalidad es dialógica? ¿Es horizontal? ¿Es crítica? ¿Es de otro tipo? ¿Es entre sujetos? ¿Es entre epistemes? En nuestra opinión, la interculturalidad se construye constantemente, es una lucha de trinchera. La existencia de este concepto no significa una guerra vencida pero sí un instrumento conceptual de lucha. El carácter propositivo que Walsh imprime sobre el término "interculturalidad crítica" lo aproxima a la transculturalidad, en el sentido de "ir más allá", de superar un determinado sistema vigente. Pero hay que definir cómo usamos el concepto de transculturalidad ${ }^{31}$ relacionado al de transdisciplinariedad.

La transdisciplinariedad es también una estrategia metodológica para lidiar con la complejidad de los fenómenos sociales; no se limita a múltiples miradas alrededor de un campo o interés científico. Ella exige desarrollos conceptuales y prácticos que ayuden a descubrir relaciones para la gran y compleja diversidad de objetos, temáticas, sujetos participantes y puntos de vista. Es un esfuerzo para obtener conocimientos que trasciendan las disciplinas, sin desconsiderarlas. La transdisciplinariedad hace relaciones entre metodologías y conceptos, aparentemente distantes y divergentes, que pueden incluir ciencia, arte, emociones y espiritualidad, humanizando, por lo tanto, la educación, las investigaciones y las

${ }^{30}$ Esta critica es desarrollada em la tesis de doctorado "Educação e o Projeto Encontro de Saberes: do Giro Decolonial ao Efetivo Giro Epistêmico", de Raoni Machado Moraes Jardim.

${ }^{31}$ Ver Carvalho y Flórez-Flórez, (2014, p.132). 
intervenciones sociales, con la posibilidad de descentrar estas prácticas del materialismo. La transdisciplinariedad es una rigurosa y exigente práctica en sus formas de investigación e intervención, y hasta cierto punto invita a la indisciplina.

En 1994, Lima de Freitas, Edgar Morin, Basarab Nicolescu, y otros intelectuales redactaron la Carta de la Transdisciplinariedad ${ }^{32}$ durante el primer Congreso Mundial transdisciplinar en Arrábia, Portugal. Llevaron al público una sistematización mordaz de la estructura académica hegemónica y propuestas de acción para modificar esta estructura. Este documento llama la atención sobre el aumento de la desigualdad entre aquellos que tienen mayor acceso al conocimiento y aquellos que tienen menos. También reconoce a la creatividad, las artes, la emoción y la espiritualidad como espacios de conocimiento. ${ }^{33}$

De forma similar, la transculturalidad implica cambios puntuales, en los sujetos, en sus epistemes y culturas. Pero el carácter dialógico de la transculturalidad no significa necesariamente una valoración innata positiva. Ninguna valoración a priori es admitida, siendo nuestra intención mayor analizar y deconstruir significados dados.

En el trabajo de Carvalho, Flórez-Flórez y Martínez (2017), la transculturalidad presente en el encuentro intercultural y dialógico entre los saberes tradicionales con los occidentales hegemónicos, puede implicar una relación de "ausencia"34, "confrontación"35 o "complementariedad"36 con las epistemes académicas. En el proyecto titulado "Encuentro de Saberes", coordinado por el profesor José Jorge de Carvalho ${ }^{37}$, los saberes tradicionales y los saberes científicos son elementos para pensar la inter y la transculturalidad, reflexionando sobre el contraste, el grado de jerarquización, y el gran cúmulo de violencia, usurpación, y exclusión de los científicos hacia los sabios tradicionales, y permite reconocer la resistencia de estos últimos. Los límites paradigmáticos y los faltantes epistémicos de nuestras instituciones académicas y científicas, se evidencian con la presencia de maestros y maestras de los saberes tradicionales, actuando como docentes e investigadores en las universidades; particularmente para la antropología en su vertiente culturalista más ortodoxa y eurocentrada.

Una forma de realizar el giro epistémico es trayendo a las universidades y a los centros de investigación a maestros y maestras de los conocimientos tradicionales, considerados los

\footnotetext{
${ }^{32}$ Ver www.filosofia.org/cod/c1994tra.htm

33 Destacamos los artículos 3, 4, 5 y 14.

${ }^{34}$ Ver Carvalho, Flórez-Flórez y Martínez (2017, p. 205).

${ }^{35}$ Ver Carvalho, Flórez-Flórez y Martínez (2017, p. 205).

${ }^{36}$ Ver Carvalho, Flórez-Flórez y Martínez (2017, p. 205/6).

${ }^{37}$ Ver Carvalho, Flórez-Flórez (2014). (C) Revista Triangulo $\quad$ Uberaba, Minas Gerai 
"otros" del conocimiento científico. Un ejemplo destacado es el proyecto de Encuentro de Saberes del Instituto Nacional de Ciencia y Tecnología para la Inclusión, dirigido por el profesor Carvalho y presente en varias universidades en Brasil y una en Colombia. Al ser los mestres protagonistas dentro de universidades y centros de investigación, el paradigma antropológico se enfrenta a su sustancia más estructural: ¿qué ocurre cuando esos "otros" hablan de sí mismos en las mismas universidades como profesores? Cuando indígenas o quilombolas, por ejemplo, cuentan juntos su propia historia, ¿están haciendo antropología? Y si ellos están hablando de los "blancos" o "no indígenas" (los "otros" de los "otros") ¿cómo enriquece y confronta esto a las disciplinas y universidades?

Este giro trae otra revolución en el paradigma académico, relacionado con el retorno de la espiritualidad diversa y la emocionalidad a la universidad, excluidas sistemáticamente por el modelo humboltiano, hegemónico en las universidades modernas del mundo occidental. La dimensión de la espiritualidad y de las emociones está presente, sin excepción, en mayor o menor medida, en todas las clases ofrecidas por los maestros y las maestras de saberes tradicionales dentro del proyecto. Esto es porque las artes y los conocimientos cultivados por las comunidades son sagrados, conectados con el mundo místico, onírico, religioso y con diversos estados de conciencia. La espiritualidad nunca salió de las comunidades tradicionales, y tampoco fue un impedimento para el desarrollo de sus ciencias, técnicas y artes (CARVALHO, FLÓREZ-FLÓREZ Y MARTÍNEZ, 2017).

Es preciso incluir a los sujetos de los conocimientos tradicionales en la enseñanza formal, es necesario crear espacio para que el diálogo horizontal e intercultural pueda ocurrir, hacia la transculturalidad de nuevos conocimientos. Cuanto mayor sean esos espacios y cuanto más alto en la jerarquía (administración, coordinación, gestión, elaboración, etc.) más efectivos serán los giros epistémicos. Este es un punto primordial para la efectividad del giro: no sólo hablar sobre los saberes tradicionales o abrir espacios puntuales para su manifestación, sino hablar con los saberes tradicionales, abriendo espacios para el protagonismo de éstos, para la alteración efectiva de las lógicas y visiones de mundo, construyendo, a partir del diálogo horizontal, nuevos conocimientos.

Para concluir, queremos resaltar que diversos académicos e intelectuales latinoamericanos, incluyendo maestros y maestras de las culturas tradicionales, han utilizado el concepto de interculturalidad tanto de forma teórica como aplicándolo a la transformación de realidades. 
El debate sobre la interculturalidad debe buscar el reconocimiento de las comunidades tradicionales y de sus epistemes. También se debe considerar la dimensión analítica de clase como elemento para las luchas de los diversos grupos sociales, una vez que la subalterización epistémica es retroalimentada por la opresión de clase que el capitalismo racista inaugura y mantiene en América. Necesitamos una revisión crítica permanente de los diferentes usos de la interculturalidad para desnaturalizar este concepto en la teoría social y en sus prácticas. La discusión sobre la interculturalidad, la inter y la transdisciplinariedad, ayuda a cualificar la educación intercultural, desarrollando reflexiones sobre la importancia de los saberes tradicionales para espacios de enseñanza pluriepistémicos, interdisciplinarios y representativos de las realidades socioculturales en América Latina.

\section{Referências}

BORÓN, A. Las ciencias sociales en la era neoliberal: entre la academia y el pensamiento crítico. XXV Congresso ALAS, 25. Ago. 2005, Porto Alegre, Brasil. Anais... Porto Alegre, 2005.

BOURDIEU, P.; WACQUANT, L. Sobre as artimanhas da razão imperialista. Revista Estudos Afro-Asiáticos. Rio de Janeiro, v. 24, n. 1, p. 15-33, 2002.

CANDAU, V. M. Educação Intercultural na América Latina: tensões atuais. In: CONGRESSO IBEROAMERICANO DE HISTÓRIA DA EDUCAÇÃO NA AMÉRICA LATINA (CIHELA), Rio de Janeiro, 2009. Anais... Rio de Janeiro: UERJ, 2009.

CARVALHO, J. J. "Espetacularização" e "canibalização" das culturas populares na América Latina. Revista Anthropológicas, Ano 14, v. 21, n. 1, p. 39-76, 2010.

CARVALHO, J. J.; FLÓREZ-FLÓREZ, J. The Meeting of Knowledges: a project for the decolonization of universities in Latin America. Postcolonial Studies, v. 17, n. 2, p. 122-139, 2014.

CARVALHO, J. J.; FLÓREZ-FLÓREZ, Juliana; MARTÍNEZ, M. El Encuentro de Saberes: Hacia una universidad pluriepistemica. In: AYALA, Nina Alejandra Cabra; RESTREPO, Camila Aschner Restrepo. Saberes nómadas: Derivas del pensamiento propio. Bogotá, Colômbia: Universidad Central. Instituto de Estudios Sociales Contemporáneos - IESCO, 2017. 
CASTRO-GOMEZ, S.; GROSFOGUEL, R. Prólogo. Giro decolonial, teoría crítica y pensamiento heterárquico. In: CASTRO-GOMEZ, S.; GROSFOGUEL, R. El giro decolonial: reflexiones para una diversidad epistémica más allá del capitalismo global. Bogotá: Siglo del Hombre Editores; Universidad Central, Instituto de Estudios Sociales Contemporáneos y Pontificia Universidad Javeriana, Instituto Pensar, 2007.

CUSICANQUI, S. R. Ch'ixinakax utxiwa: una reflexión sobre prácticas y discursos descolonizadores. Buenos Aires: Tinta Limón, 2010.

FREIRE, P. Pedagogia do oprimido. 17. ed. Rio de Janeiro: Paz e Terra, 1987.

FALS BORDA, O. Brasil: Campesinos y Vivienda. Bogotá: Imprenta Nacional, 1963.

FALS BORDA, O. Por la praxis: el problema de cómo investigar la realidad para transformarla. Servico Social e Sociedade, No. 11, Sao Paulo, Cortez, 1982.

FALS BORDA, O.; MORA-OSEJO, L. E. La superación del eurocentrismo, Enriquecimiento del saber sistémico y endógeno sobre nuestro contexto tropical, 2002, Polis, Revista de la Universidad Bolivariana, vol. 2, núm. 7, p. 0 Universidad de Los Lagos, Santiago, Chile, 2004.

INSTITUTO BRASILEIRO DE GEOGRAFIA E ESTATÍSTICA - IBGE. Síntese de Indicadores Sociais: uma análise das condições de vida da população brasileira. 2016. Disponível em: < https://biblioteca.ibge.gov.br/visualizacao/livros/liv98965.pdf>. Acesso em: 20 jun. 2018.

LANDER, E. Ciências Sociais: saberes coloniais e eurocêntricos”. In: LANDER, E. (Org.). A colonialidade do saber: eurocentrismo e ciências sociais. Perspectivas latino-americanas. Buenos Aires: CLACSO, set. 2005. (Coleção Sur Sur).

MALDONADO-TORRES, N. Sobre la colonialidad del ser: contribuciones al desarrollo de un concepto. In: CASTRO-GÓMEZ, S.; GROSFOGUEL, R. El giro decolonial: reflexiones para una diversidad epistémica más allá del capitalismo global. Bogotá: Siglo del Hombre Editores, Universidad Central, Instituto de Estudios Sociales Contemporáneos y Pontificia Universidad Javeriana, Instituto Pensar, 2007.

MALDONADO-TORRES, N. "Sobre la colonialidad del ser: contribuciones al desarrollo de un concepto". In: CASTRO-GÓMEZ, S. \& GROSFOGUEL, R. (Coords.) EI giro decolonial: reflexiones para una diversidad epistêmica más allá del capitalismo global. Bogotá: Siglo del Hombre Editores; Universidad Central, Instituto de Estudios Sociales Contemporáneos, Pontificia Universidad Javeriana, Instituto Pensar, 2007. 
MATO, D. Interculturalidad e inclusión en las universidades. Diferencias culturales, de modos de producción de conocimiento y de modalidades de aprendizaje”. In: MATO, D. (Coord.). Diversidad Cultural e Interculturalidad en Educación Superior: Experiencias en América Latina. Caracas: IESALC-UNESCO, 2008; 2006.

MATO, D. No “estudiar al subalterno", sino estudiar con grupos sociales "subalternos" o, al menos, estudiar articulaciones hegemónicas de poder”. Desafios. Bogotá, Colômbia, p. 237$264,2014$.

PAYNE, M. Diccionario de Teoría Crítica y Estudios Culturales. Buenos Aires: Paidós, 2008.

WALSH, C. Interculturalidad y colonialidad del poder. Un pensamiento y posicionamiento “otro" desde la diferencia colonial. In: CASTRO-GÓMEZ, S.; GROSFOGUEL, R.. El giro decolonial: reflexiones para una diversidad epistémica más allá del capitalismo global. Bogotá: Siglo del Hombre Editores; Universidad Central, Instituto de Estudios Sociales Contemporáneos y Pontificia Universidad Javeriana, Instituto Pensar, 2007.

WALSH, C. Interculturalidad y (de)colonialidad: perspectivas críticas y políticas. Visão Global, Joaçaba, v. 15, n. 1-2, p. 61-74, 2012.

ZIZEK, S.; JAMESON, F.; GRUNER, E. Estudios Culturales - Reflexiones sobre el Multiculturalismo. Ed. Paidós, 2008. 